\section{GENE DELIVERY}

\section{Magnetic delivery}

Zhu, H. et al. Nat. Biomed. Eng. doi:10.1038/

s41551-018-0318-7 (2018)

Need to get your gene editor to a specific tissue or organ in vivo? Magnets might do the trick, says a team of bioengineers from Rice University in Houston, Texas.

The researchers developed a novel delivery vehicle for a gene editor that consists of an inactivated baculoviral vector combined with magnetic nanoparticles. The vector is derived from a virus that infects the alfalfa looper moth and can efficiently carry large cargo, like lengthy CRISPR/ Cas9 sequences, while the magnets can influence deep tissues without adverse effect. Once in the body (injected, in a mouse's case, intravenously via the tail), you need only apply a small magnet near the desired editing site to release the editor there. The researchers demonstrate the system's in vivo potential in mouse liver, spleen, and subcutaneous tumors. EPN

https://doi.org/10.1038/s41684-018-0234-1

\section{IMAGING TECHNIQUES}

\section{Magnetic rotations}

Berndt, F. et al. Nat. Commun. 9, 5025 (2018)

To image a living sample under a light-sheet microscope, the specimen needs to be fixed and kept still. But a single view might not reveal all the detail desired. A new approach uses magnets to add a little rotation. Small magnetic agarose spheres can be embedded in the agarose that holds the sample itself, or in the case of animals like zebrafish that develop in a fluid-filled chorion, directly into the yolk. A simple magnet or, for more dynamic three-dimensional control, a set-up of electromagnets can then be used to re-orient the sample under the microscope.

The researchers apply the new magnetic approach in $6 \mathrm{~mm}$ Artemia crustaceans and $12 \mathrm{~mm}$ mouse embryos, zebrafish embryos (which rotate within their chorion), and zebrafish larvae held in a glass capillary.

\section{BIOLUMINESCENCE IMAGING}

\section{Beneath the glowing yeast}

Kotlobay, A.A. et al. PNAS. 115, 12728-12732 (2018)

Natural and engineered bioluminescent systems have been tapped by scientists to provide non-invasive imaging in vivo. But the complete biochemical details involved in the approximately 40 known natural systems aren't that well known-only one cascade has been fully described from starting metabolite to luciferin, the substrate that interacts with an enzyme to produce light. It's found in bacteria, a pretty distant genetic relevant from animals.

But move over, bacteria, there's a fungal version now to consider. A team of Russian researchers recently identified and described all the enzymatic steps that lead to the green glow of the eukaryotic yeast Pichia pastoris. They demonstrate its potential as an in vivo reporter in other eukaryotes, testing its function in Xenopus laevis embryos and tumor xenografts in mice. $E P N$

https://doi.org/10.1038/s41684-018-0236-Z

\section{NEUROSCIENCE \\ Macaque vs. man transcriptomes}

Zhu, Y. et al. Science 362, eaat8077 (2018)

The nervous system of a macaque is more closely related to a human's than that of a mouse. Despite the similarities, nonhuman primates are not human. A new study provides a new resource that measures both convergence and divergence between macaque and man as their brains develop.

The researchers recorded single-cell transcriptome data at in several different tissues at several developmental time points for each species, as well as in samples from chimpanzees. They then developed an algorithm to analyze transcriptomic signatures over time. Their results suggest divergence is greatest during prenatal development and after adolescence. They also observe a number of temporal and spatial differences in the human brain that may underpin a variety of neurological and psychiatric diseases. $E P N$

https://doi.org/10.1038/s41684-018-0237-y

Ellen P.Neff

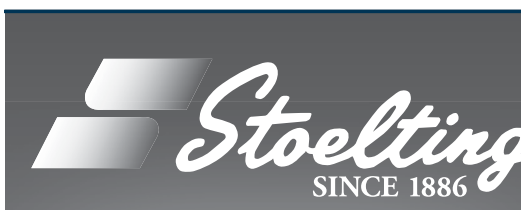

www.StoeltingCo.om

Lab Animal Identification
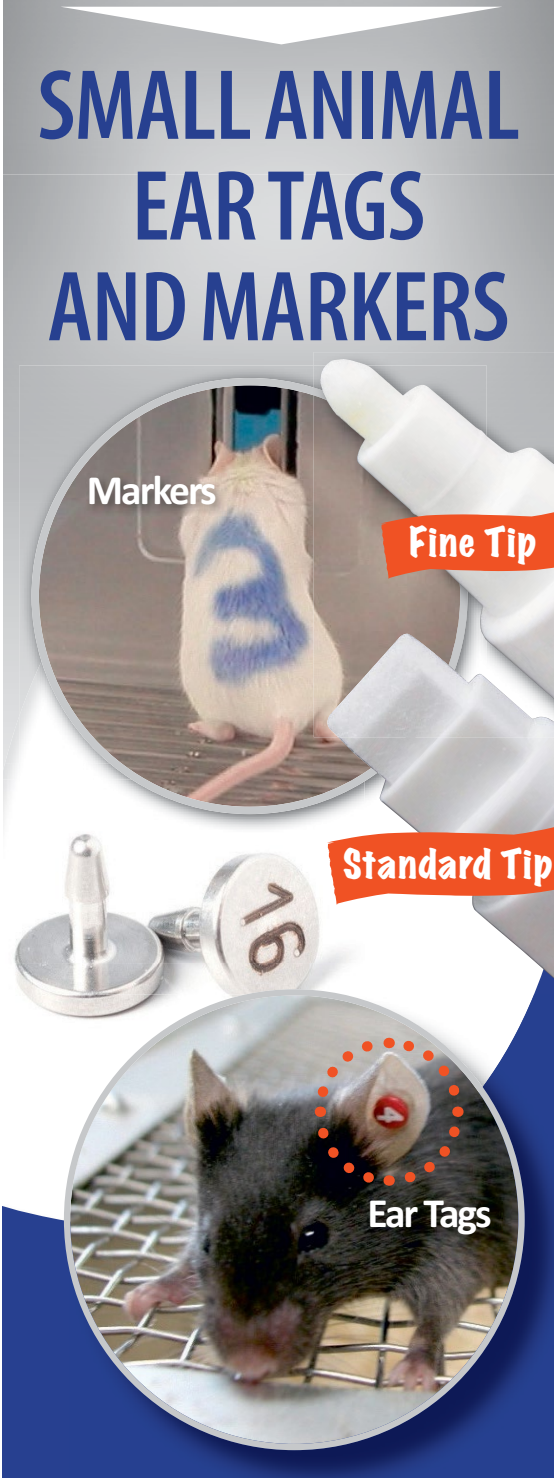

Ear Tag and Marker Features:

- Quick and easy identification

- Long-lasting

- Nontoxic, non-hazardous

- Multiple colors options available

Samples now available,

Contact Stoelting for

more details!

info@stoeltingco.com 